\title{
Effect of Glycated Hemoglobin Induced Lipid Peroxidation on Membrane Bound Acetyl Cholinesterase
}

\author{
N. Jeyashanthi*, P. Ravikumar and N. N. Baalakumar \\ Department of Biomedical Engineering, Velalar College of Engineering and Technology, Erode - 638012, India; \\ njeyashanthi@gmail.com
}

\begin{abstract}
This study examines the relationship between erythrocyte membrane bound Acetyl cholinesterase (AChE) activity, glycated hemoglobin ( $\mathrm{HbA1}$ ) level and lipid peroxidation (formation of malondialdehyde). The aim was to elucidate the effect of $\mathrm{HbA} 1 \mathrm{c}$ and lipid peroxidation on the activity of acetyl cholinesterase (AChE). Control subjects were non diabetic volunteers and the test group was diabetic patients with fasting glucose level of more than $150 \mathrm{mg} \%$. HbA1c, total hemoglobin, plasma glucose, lipid peroxidation and activity of AChE were determined. Observations indicate that elevated levels of lipid peroxidation in erythrocyte membrane of glycated hemoglobin of diabetic patients. HbA1c significantly increases lipid peroxidation and decreases the activity of AChE. Our data indicates that erythrocyte membrane bound enzyme AChE is significantly related to lipid peroxidation. Glycation of the hemoglobin increases the lipid peroxidation and alters the membrane bound enzyme function.
\end{abstract}

Keywords: Glycosylation, Malondialdehyde MDA, AChE, HbA1c

\section{Introduction}

Diabetes mellitus is a disorder of carbohydrate metabolism in which the ability to oxidize and utilize carbohydrates is lost as a result of defects in insulin secretion, insulin action, or both (WHO, 1999). It is characterized by hyperglycemia and disturbances of carbohydrate, fat and protein metabolism. In diabetes, Glycated hemoglobin $(\mathrm{GHb})$ is formed by a nonenzymatic post translational modification reaction which involves the reaction of glucose with valine and lysine residues in haemoglobin ${ }^{1}$.

The human erythrocytes are freely permeable to glucose and within each erythrocyte glycated hemoglobin is formed continuously from haemoglobin. Glycated hemoglobin plays an important role in monitoring the long term blood glucose of diabetics ${ }^{2}$. Nonenzymatic glycation is a spontaneous chemical reaction in which labile Schiff's bases and more stable Amadori products are formed. Advanced glycation end products (AGEs) are then formed through oxidative reactions and cause irreversible chemical modifications of proteins ${ }^{3}$.

Free radicals are produced as a result of glycosylation of several proteins including hemoglobin $(\mathrm{Hb})$ by non-enzymatic mechanisms ${ }^{4,5}$. Subsequently free radicals change lipid/protein ratio of membranes by affecting polyunsaturated fatty acids. Lipid peroxidation is the oxidative deterioration of polyunsaturated fatty acids. Lipid peroxidation produces aldehydes, superoxide, hydrogen peroxide, hydroxyl radicals, and chemical substances like malondialdehyde (MDA). MDA is a significant end product of LPO that is used normally as a marker of lipid peroxidation ${ }^{6}$.

By-products of lipid peroxidation have been shown to cause profound alterations in the structural organization and functions of the cell membrane including decreased membrane fluidity, increased membrane permeability,

${ }^{*}$ Author for correspondence 
inactivation of membrane-bound enzymes and loss of essential fatty acids ${ }^{7}$. Erythrocyte membrane is more vulnerable to lipid peroxidation due to constant exposure to high oxygen tension and richness in polyunsaturated fatty acid, respectively.

The objective of this work is to estimate the oxidative stress in diabetic patients and assess the relationship between the hyperglycemia and glycated hemoglobin levels. The diabetic state was evaluated by determining the fasting blood sugar and glycosylated hemoglobin and the level of oxidative stress was estimated by quantifying the thiobarbituric acid reacting substance. The effect of TBRS on the activity of erythrocyte membrane bound enzyme was studied by assaying the activity AChE.

\section{Materials and Methods}

\subsection{Sample Collection}

This study contained two groups: control and test. Control group were nondiabetic individuals, and twenty diabetic patients with fasting glucose level of more than $150 \mathrm{mg} \%$ were taken as test group.

\subsection{Estimation of Glycated Haemoglobin by Affinity Chromatography}

Glycated haemoglobin was separated from the nonglycated fraction by affinity gel columns (Glycogel B). The absorbance (A) of each fraction was measured at $414 \mathrm{~nm}$ and calculated the percentage of glycosylated haemoglobins according to the formula ${ }^{8}$ :

$$
\% \text { glyHb }=\frac{5 \times \text { A bound }}{\left(20.1 \times A_{\text {not bound }}\right)+\left(5 \times A_{\text {bound }}\right)} \times 100
$$

\subsection{Estimation of Malondialdehyde (MDA)}

The concentration of malondialdehyde in erythrocyte was estimated by the procedure given by Jain et $\mathrm{al}^{15}$. The blood hemoglobin level was determined by the method of Drabkin?.

\subsection{Isolation of Erythrocytes and Membrane Preparation}

Red blood cell acetylcholinesterase (AChE) is an integral membrane protein which hydrolyzes acetylcholine. Being an erythrocyte outer layer membrane protein, its function (catalytic capacity) might be influenced directly by enzymatic effectors and indirectly by membrane phospholipid or cytoskeleton modulation ${ }^{10}$. This enzyme can be used as a marker of membrane integrity and to evaluate the effect of TBRS on membrane bound enzyme. Erythrocyte AChE activity was determined by Kaplan's modified method ${ }^{11}$.

\section{Results and Discussion}

The diabetic state was confirmed by high fasting blood glucose level in test group and the results are depicted in Figure 1 and Table 1.

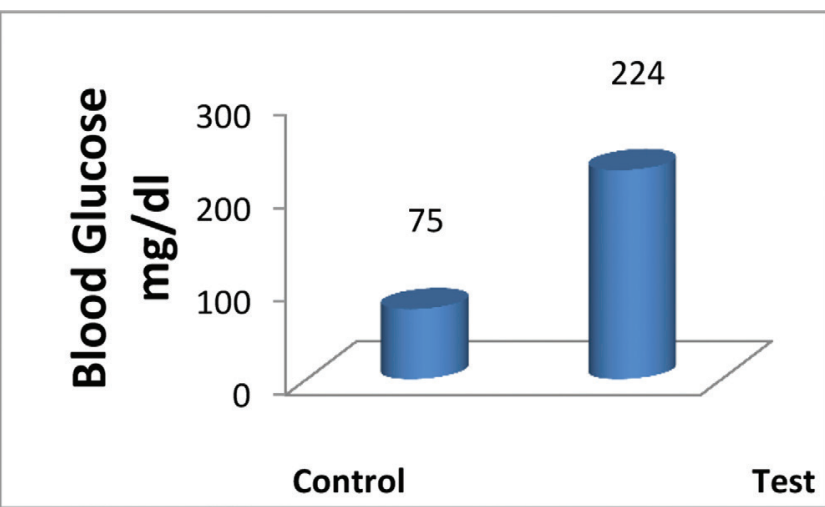

Figure 1. Fasting blood glucose level.

The erythrocytes were isolated from the both individuals with diabetes mellitus (test) and normal healthy individuals (control) for determination of TBARS. The results are given in Figure 2 and Table 1.

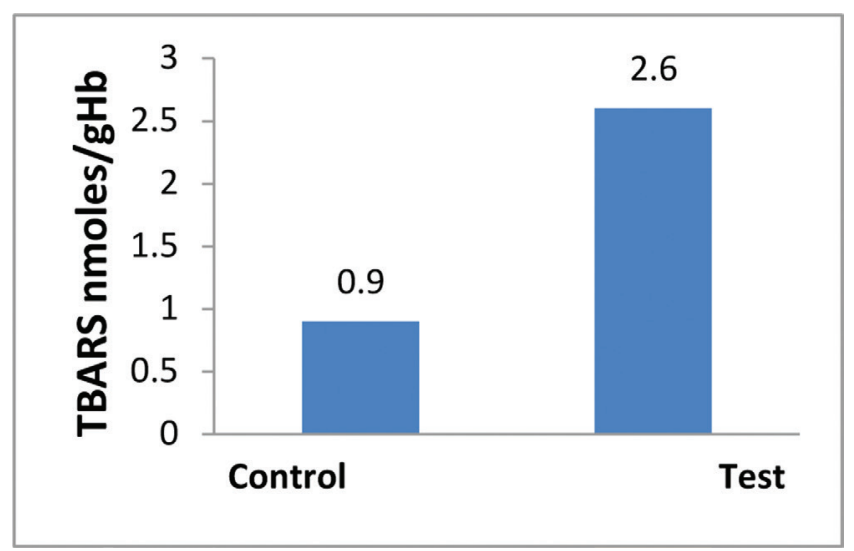

Figure 2. TBARS level in normal and diabetic state. 
Erythrocyte TBARS level was higher in diabetes $(2.6$ $\pm 0.6 \mathrm{mmoles} / \mathrm{gHb})$ than the controls $(0.9 \pm 0.01 \mathrm{mmoles} /$ $\mathrm{gHb}$ ). The estimated amount of glycated haemoglobin was shown in Figure 3 and Table 1. The glycated haemoglobin level was higher in diabetes $(9.7 \pm 1.2 \mathrm{mmole} / \mathrm{g}$ of $\mathrm{Hb})$ than the controls $(6.2 \pm 0.9 \mathrm{mmole} / \mathrm{g}$ of $\mathrm{Hb})$.

The fasting blood sugar concentration has no correlation with erythrocyte TBARS level but a positive correlation between the fasting blood sugar concentration and the glycated haemoglobin level was observed in normal and test group.

There is a significant $(\mathrm{p} \geq 0.05)$ increase in the TBARS level in the diabetic erythrocytes when compared to the normal erythrocytes.

Table 1. FBS, TBARS, Glycated Hemoglobin levels and $\mathrm{AChE}$ in diabetes and control group

\begin{tabular}{|l|l|l|}
\hline Parameters & Control & Diabetes \\
\hline Fasting blood (mg \%) & $75 \pm 3.4$ & $224 \pm 9.7$ \\
\hline TBARS (mmoles/gHb) & $0.9 \pm 0.01$ & $2.6 \pm 0.6$ \\
\hline $\begin{array}{l}\text { Glycated hemoglobin (mmole/g } \\
\text { of } \mathrm{Hb})\end{array}$ & $6.2 \pm 0.9$ & $9.7 \pm 1.2$ \\
\hline AChE (U) & $2.8 \pm 0.4$ & $1.2 \pm 0.3$ \\
\hline
\end{tabular}

Acetyl choline activity expressed as millimoles of ACh hydrolyzed/min/mg protein.

Values are mean $\pm S D$ of 20 observations.

Oxidative stress is a condition in which the concentration of free radicals and Reactive Oxygen Species (ROS) is increased. In normal physiological conditions these free radicals and ROS have been removed by antioxidant system, but become toxic when not being removed by the antioxidant system. The formation of ROS is increased in

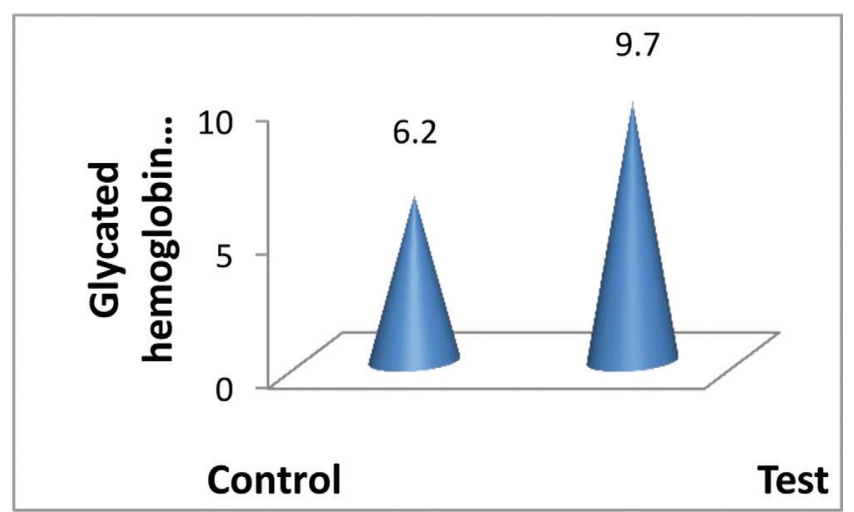

Figure 3. Level of glycated hemoglobin.
Type I and Type II diabetes mellitus. The secondary complications in diabetes patients are directly associated to the increased oxidative stress. Free radical induced lipid peroxidation is a major cause for the development secondary complications in diabetes mellitus. The sources of increased concentration of free radicals in diabetic condition are: increased production of reactive oxygen species, mainly from glycated proteins or lipid peroxidation, autooxidation of glucose and decreased activity of antioxidant systems ${ }^{12}$.

In this study the diabetic patients showed significant increase in lipid peroxidation level. It was observed by the increased levels of TBARS in diabetic patients. The increased levels of TBARS in diabetes mellitus may be due to the increased concentration of free radicals which might be due to increased glycosylation of proteins. The glycosylated proteins could form a source for free radicals. There is a significant correlation between lipid peroxidation and blood glucose level in diabetes mellitus. The increased level of blood glucose results in increased lipid peroxidation, enolisation of glucose and reduction of $\mathrm{O}_{2}$. Molecular oxygen is reduced to form $\alpha$ - ketoaldehydes, hydrogen peroxide and ROS. Hydrogen peroxide produced during the above reactions produces hydroxyl radicals. The ROS leads peroxidation of fatty acids in membrane and accumulation of TBARS ${ }^{13}$.

This study was conducted to know the correlation between blood glucose level and glycosylation of haemoglobin by determining the glycated haemoglobin levels. There was a significant $(\mathrm{P} \leq 0.05)$ increase in the level of glycated haemoglobin in diabetic patients. The concentration of glycated haemoglobin is proportional to blood glucose levels. Diabetic patients with increased concentrations of blood glucose invariably contain increased concentration of glycated haemoglobin ${ }^{14}$.

Results of the study also suggest that an increased TBARS level is linked to the increased level of glycated haemoglobin. Several studies have reported similar results (Gallan MP to Konukoglu). The glycated haemoglobin is an indicator of blood glucose level of diabetic patients over previous few months (2-3 months) and TBARS was considered as an indicator for assessing the oxidative state in diabetes mellitus. The glycosylation mediated structural changes in hemoglobin is one of the important factor for increased lipid peroxidation in diabetes. Jain et al. (Jain SK) have also reported a positive correlation between glycated haemoglobin and TBARS level. 
Increased glycosylation of haemoglobin $(\mathrm{Hb})$ in diabetic state induces the synthesis of Free radicals by non-enzymatic mechanisms ${ }^{10}$. The highly reactive free radicals may lead to oxidation injury to the living cells by altering the structure and state of macromolecules including lipids, carbohydrates, proteins and nucleic acids ${ }^{15}$. The lipid/protein ratio of membranes is affected by free radicals. It is potentially harmful because its uncontrolled self-enhancing process causes disruption of membranes, lipids and other cell components ${ }^{16}$.

The results of this study show (Figure 4 and Table 1) that AChE activity RBCs were decreased significantly in diabetic patients as compared with the control group.

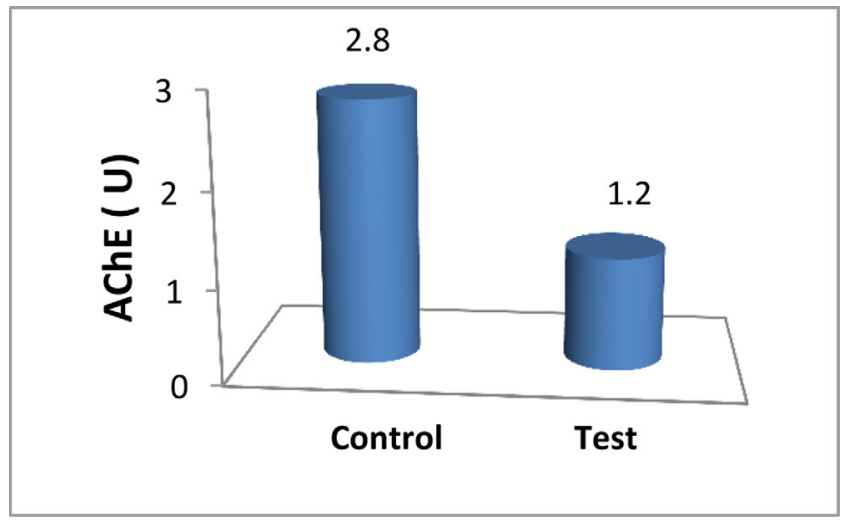

Figure 4. The activity of erythrocyte membrane bound AChE.

Several studies have also demonstrated marked reduction in cholinergic function in diabetic state ${ }^{17}$. Acetylcholinesterase is a specific choline esterase, hydrolyzing predominantly choline esters, and characterized by high concentrations in brain, nerve and red blood cells $(\mathrm{RBCs})^{18}$. It is responsible for interrupting normal nerve transmission at the synapse by hydrolyzing the neurotransmitter Acetyl choline to acetic acid and choline. This transmission interruption prevents unnecessary stimulation of nervous system.

Acetylcholinesterase is a membrane bound protein attached in the lipid bilayer of the membrane by a small hydrophobic moiety, possibly through phosphatidylinositol. The activity of the enzyme Acetylcholinesterase may be changed by the hydrophobic surroundings (membrane lipid bilayer) of the enzyme ${ }^{19}$. Previous researches also accounted a significant hyperpolarization of red blood cell membranes of diabetic patients ${ }^{20}$. The modifications in erythrocyte membrane properties, namely membrane lipid bilayer packing and composition, fluidity and membrane potential, during diabetes can decrease the activity of the enzyme acetylcholinesterase ${ }^{21}$.

The earlier studies suggest that uncontrolled diabetes is associated with significant alterations in the cholinergic systems ${ }^{17}$.

In conclusion the determination TBRAS and glycated hemoglobin in diabetic patients would be used to review the state of diabetic patients. Improvement of glycemic control will decrease the oxidative stress in diabetic patients. Prevention of oxidative stress may help to maintain the membrane integrity and prevent the development of secondary complications in diabetic patients.

\section{References}

1. Chandalia HB, Krishnaswamy PR. Glycated hemoglobin. Current Science. 2002; 83:1522-31.

2. Gallan MP, Carrascosa A, Gussinye M, Dominguez C. Biomarkers of diabetes associated oxidative stress and antioxidant status in young diabetic patients with or without subclinical complications. Free Radical Biology \& Medicine. 2003; 34(12):1563-74. https://doi.org/10.1016/S0891-5849(03)00185-0.

3. Dominguez M, Wasserman JD, Freeman M. Multiple functions of the EGF receptor in Drosophila eye development. Current Biology. 1998; 8(19):1039-48. https://doi.org/10.1016/S0960-9822(98)70441-5.

4. Signal PK, Bello-Klein A, Farahmand $\mathrm{F}$ and Sandhawalia V. Oxidative Stress and Functional Deficit in Diabetic Cardiomyopathy. Advances in Experimental Medicine and Biology. 2001; 498:213-20. https://doi.org/10.1007/978-1-4615-1321-6_27.

5. Mercuri F, Quagliaro L and Ceriello A. Oxidative Stress Evaluation in Diabetes. Diabetes Technology \& Therapeutics. 2000; 2(4):589-600.

6. Jacob RA and Burri BJ: Oxidative damage and defense. The American Journal of Clinical Nutrition. 1996; 63:985-90. https://doi.org/10.1093/ajcn/63.6.985.

7. Van Ginkel G, Sevanian A. Lipid peroxidation induced membrane structural alterations. Methods in Enzymology. 1994; 233:273-88. https://doi.org/10.1016/S0076-6879(94) 33031-X.

8. Weiner K. Tests in disorders of glucose metabolism. Gowenlock AH editor Varley's practical clinical biochemistry, 6th ed. New Delhi: CBS Publisher. 1988; p. 333-49.

9. Drabkin DL, Austin JM. Spectrophotometric constants for common hemoglobin derivatives in human, dog and rabbit blood. The Journal of Biological Chemistry. 1932; 98:719-33. 
10. Karatas F, Halifeoglu I, Karatepe M, Konar V, Canatan H, Colak R. Evaluation of changes in levels of serum selenium, MDA and antioxidant vitamins (A, E, C) in diabetic patients. Aralik 2006; 20(6):391-5.

11. Kaplan E, Herg F and Hsu KS. Erythrocyte acetylcholinesterase activity in $\mathrm{ABO}$ emolytic disease of the newborn. Pediatrics. 1964; 33:205 PMid:14117375.

12. Stephen SM Chung, Eric CM Ho, Karen SL Lam and Sookja K Chung. 2003 Contribution of Polyol Pathway to DiabetesInduced Oxidative Stress. Journal of American Society of Nephrology. 2003; 114:S233-6.

13. Jain SK, Mc Vie R, Duet J, Herbst JJ. Erythrocyte membrane lipid peroxidation and glycosylated haemoglobin in diabetes. Diabetes.1989; 38:1539-43. https://doi.org/10.2337/diabetes.38.12.1539. https://doi. org/10.2337/diab.38.12.1539. PMid:2583378.

14. Gabby KH, Sosenko JM, Banuchi GA, Minin MJ, Fluckigu R. Glycosylated hemoglobins; increased glycosylation of haemoglobin A in diabetes patients. Diabetes. 1979; 28(4):337-40. https://doi.org/10.2337/diab.28.4.337. https://doi.org/10.2337/diabetes.28.4.337.

15. Vadde R, Jailkhani R. Evaluation of oxidative stress in insulin dependent diabetes mellitus (IDDM) patients. Diagnostic Pathology. 2007; 2:22. https://doi.org/10.1186/1746-15962-22. PMid:17603912 PMCid:PMC1936413.
16. Mahboob M, Rahman MF, Groover P. Serum lipid peroxidation and antioxidant enzyme levels in male and female diabeticpatients. SingaporeMedicalJournal.2005;46(7):322. PMid:15968442.

17. Agarwal VR, Rastogi AK, Sahib MK and Sagar P. Acta Diabetologica. 1985; 22:359-63.

18. Kaplay SS Acetylcholineesterase and butyrylcholineesterase of developing human brain. Biology of the Neonate. 1976; 28:65-73. https://doi.org/10.1159/000240805.

19. Ott P. Membrane acetylcholinesterases: purification, molecular properties and ... Biochimica et Biophysica Acta. 1985; 822:375-92. https://doi.org/10.1016/0304-4157(85)90016-4.

20. Augustyniak K, Zavodnik I, Palecz D, Szosland K \& Bryszewska $\mathrm{M}$. The effect of oxidizing agent and diabetes mellitus on the human red blood cell membrane potential. Clinical Biochemistry 1996; 29:283-6. https://doi. org/10.1016/0009-9120(95)02045-N.

21. Kolling A, Maldonada C, Ojeda F and Dieht HA. Membrane fluidity of microsomal and thymocyte membranes after $\mathrm{x}$-ray and UV irradiation. Radiation and Environmental Biophysics. 1994; 33:303-13. https://doi.org/10.1007/BF01210452. PMid:7708904.

22. Agarwal VR, Rastogi AK, Sahib MK and Sagar P. Acta Diabetologica. 1985; 22:359-63. 